\title{
Kinematics of gait in Golden Retriever Muscular Dystrophy
}

\author{
Anthony P. Marsh ${ }^{a}{ }^{,}$, Joel D. Eggebeen ${ }^{a}$, Joe N. Kornegay ${ }^{b}$, Chad D. Markert ${ }^{c}$, and Martin K. \\ Childers $^{\mathrm{C}}$ \\ Anthony P. Marsh: marshap@wfu.edu

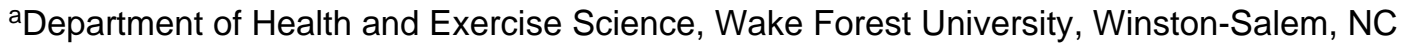 \\ 27109, United States \\ ${ }^{b}$ Departments of Pathology and Laboratory Medicine and Neurology, School of Medicine, \\ University of North Carolina-Chapel Hill, Campus Box 7525, Chapel Hill, NC 27599-7525, United \\ States \\ 'Neurology and Regenerative Medicine, Wake Forest University Health Sciences, Medical Center \\ Blvd., Winston-Salem, NC 27157, United States
}

\begin{abstract}
The goal of this study was to quantify the two-dimensional kinematics of pathologic gait during overground walking at a self-selected speed at the stifle (knee) and hock (ankle) joints in six Golden Retriever Muscular Dystrophy (GRMD) dogs and six carrier littermates (controls). We found that GRMD dogs walked significantly slower than controls $(p<0.01)$. At the stifle joint, both groups displayed similar ROM (range of motion), but compared to controls, GRMD dogs walked with the stifle joint relatively more extended. At the hock joint, GRMD dogs displayed less ROM (range of motion) and walked with the joint relatively less flexed compared to controls. We controlled for gait speed in all analyses, so the differences we observed in joint kinematics between groups cannot be attributed solely to the slower walking speed of the GRMD dogs. This is the first kinematic study of gait in the GRMD dog, an important step in using this model in preclinical trials.
\end{abstract}

\section{Keywords}

Muscular dystrophy; Locomotion; Biomechanics; Canine; Physical function

\section{Introduction}

Duchenne muscular dystrophy (DMD) affects approximately 1 in 3500 males born each year worldwide [1], resulting in devastating weakness, contractures, and early death. Despite over a century of research, there is no cure for this X-linked inherited disease, stemming, in part, from the lack of animal models that reflect both the genotype and phenotype of DMD patients. However, a canine model of DMD, termed Golden Retriever Muscular Dystrophy (GRMD), reflects both the genotype and phenotype of DMD. Affected dogs suffer rapidly progressive, fatal decline strikingly similar to the human condition [2-7]. Unlike the dystrophin-deficient $m d x$ mouse, which exhibits a relatively mild phenotype, GRMD dogs display a severe phenotype, particularly in limb, respiratory, and heart muscles, such that by 8 months of age the majority of affected dogs manifest significant gait abnormalities. 
Gait analysis in dogs has received an increasing amount of attention as a way to quantify both musculoskeletal diseases and also to assess the efficacy of clinical interventions [8]. Kinematic and kinetic analyses in dogs have been conducted using over-ground [9-15] and treadmill walking [16-20]. However, we are not aware of any published studies that have conducted a kinematic evaluation of gait in the GRMD model. Characterizing the functional phenotype of GRMD is essential if it is to be used as a clinically relevant endpoint to examine the efficacy of novel treatments of DMD, such as stem cell or gene therapy, alone or in combination with physical exercise. The goal of the present investigation was to quantify pathologic gait during over-ground walking in GRMD dogs using a twodimensional kinematic analysis focused on the stifle and hock angles of the pelvic limb. We studied two groups: GRMD dogs and their age-matched carrier littermates heterozygous for the disease. We tested the hypothesis that GRMD dogs walk significantly slower than their carrier littermates. We also tested the hypothesis that compared to age-matched controls, stifle and hock joints demonstrate less range-of-motion (ROM) in GRMD dogs.

\section{Methods}

\subsection{Dogs}

Golden retriever cross-bred dogs (6 males, 6 females) from a GRMD colony were studied. Dogs were used and cared for according to principles outlined in the National Institutes of Health Guide for the Care and Use of Laboratory Animals Newborn GRMD dogs were identified based on elevation of serum creatine kinase and subsequently developed characteristic clinical signs. Genotype was confirmed by polymerase chain reaction (PCR) methodology as described previously [21]. Prior to data collection, all dogs had a complete physical, orthopedic, and neurologic assessment to ensure that there were no underlying conditions that would influence gait.

\subsection{Video collection}

Prior to filming, retro-reflective markers ( $3 \mathrm{M}$ Scotchlight, $3 \mathrm{M}$, St Louis, MO) were placed on anatomical landmarks of the right and left pelvic limbs by one of the authors (JNK). The landmarks were the greater trochanter of the femur (hip joint), a point equidistant between the lateral epicondyle of the femur and the fibular head (stifle joint), the lateral malleolus of the distal tibia (tarsal joint), and the distolateral aspect of the fifth metatarsus (metatarsophalangeal joint). Manual flexion and extension of the joints after application of the markers was used to verify that the marker positions were as close as possible to the joint centers. To facilitate marker placement, hair over the hindquarters was clipped and glue was used to fix the markers (Vetbond Tissue Adhesive, 3 M, St. Paul, MN). Following marker application, each dog was familiarized to the filming environment.

Digital video (DCR-TRV 18, Sony, Japan) of sagittal plane motion was collected at $30 \mathrm{~Hz}$ during over-ground walking at the dog's self-selected pace (Fig. 1) with a single camera. Gait speed was measured by timing the dog using a stopwatch over a $2.4 \mathrm{~m}$ distance in the sagittal plane within the camera's field of view. The filming location incorporated $3 \mathrm{~m}$ before and $3 \mathrm{~m}$ after the filming region to allow the dogs to accelerate to a constant speed with a consistent gait pattern prior to data collection and to slow down well past the filming field of view. We aligned the walking path of the dogs to the wall that created the backdrop to the filming environment. Next, tape was placed on the floor to define the distance that was used to time the gait speed and the plane of the path of progression. If the dog deviated from this plane the trial was not used for analysis. We used a bubble level to level the camera so that the axis of the lens was horizontal. The camera was positioned $8 \mathrm{~m}$ from the plane of progression with the axis of the lens perpendicular to the plane, and zoomed to capture the entire walking field $(2.4 \mathrm{~m})$ within the camera field of view. 
During filming the dogs were led on a leash by the same experienced handler. Data were collected on both sides of each dog. A record was maintained regarding the quality of each gait pass ranked by three observers (APM, JMK, MKC). Due to the clinical signs of GRMD (weakness, lethargy, shortness of breath), affected dogs were encouraged with praise, an occasional leash tug, and a food reward to coax them through the filming area at their selfselected pace. Because brief periods of activity were challenging for GRMD dogs, rest was provided as needed. This procedure was repeated for age-matched carrier controls.

\subsection{Video processing}

Trials that represented a consistent, self-paced gait within the calibrated plane as identified by three of the investigators were selected for digitizing. Trials where dogs increased or decreased speed, stopped, or walked out of the calibrated sagittal plane were excluded from analysis. Three to four complete gait cycles were visible on each pass. A complete gait cycle was defined as starting at initial pelvic limb ground contact and ending with ipsilateral pelvic limb ground contact. Two trials for the right and left side of each dog were identified for further analysis.

Digitizing software (VideoExpert, Motion Analysis Corp., Santa Rosa,CA) and MATLAB (The Math Works, Natick, MA)programs were used to calculate the joint angles of the stifle (knee) and hock (ankle). The three points used to operationally define the stifle joint were the centers of the reflective markers, which were placed on the three following landmarks: the greater trochanter of the femur (hip joint), a point equidistant between the lateral epicondyle of the femur and the fibular head (stifle joint), and the lateral malleolus of the distal tibia (tarsal joint). The three points used to operationally define the hock joint were the centers of the reflective markers placed at a point equidistant between the lateral epicondyle of the femur and the fibular head (stifle joint), the lateral malleolus of the distal tibia (tarsal joint), and the distolateral aspect of the fifth metatarsus (metatarsophalangeal joint). Raw $x-$ $y$ co-ordinate data were filtered using a Butterworth filter with a cutoff frequency of $4 \mathrm{~Hz}$. Each gait cycle was normalized in time (100\% of gait cycle) with complete cycles averaged together for each side of the body. Maxima and minima for each complete cycle were recorded and joint range-of-motion (ROM) calculated for each joint.

\subsection{Statistical analysis}

Statistical software (SPSS 16; SPSS Inc., Chicago, IL) was used for all analyses. Given the exploratory nature of this study, $p<0.1$ was considered significant. Descriptive statistics (mean $\pm \mathrm{SD}$ ) were calculated for all variables. A three way repeated measures analysis of variance (RMANOVA) with one between factor (group: GRMD, carrier) and two within group factors (trial: 1, 2; side: right, left) was used to examine gait speed data. Fisher Least Significant Difference post hoc testing was used to determine if significant differences existed between trials or between the right and left sides. A three factor analysis of covariance (ANCOVA), using gait speed as the covariate, was used to examine the minimum and maximum angles, and ROM, at the stifle and hock joints.

\section{Results}

\subsection{Subject demographics}

The average age, shoulder height and body mass of dogs was $25.9 \pm 8.8$ months, $47.3 \pm 3.5$ $\mathrm{cm}$; and $18.7 \pm 3.1 \mathrm{~kg}$, respectively (Table 1 ).

\subsection{Gait speed}

GRMD dogs walked significantly $(p<0.01)$ slower than their carrier littermates $(1.18 \pm$ 0.34 and $1.80 \pm 0.49 \mathrm{~m} / \mathrm{s}$, respectively). There were no significant 3-way or 2-way 
interactions (3-way RMANOVA). There were no detectable differences with respect to trial or side. That is, gait speeds of trials from both the right and left sides were not different. The mean within dog coefficient of variation (CV) for gait speed was $5.1 \%$ and $5.5 \%$ for the affected and unaffected dogs, respectively. Therefore, gait speed was averaged and subsequently used in the ANCOVA analyses presented below.

\subsection{Joint angles}

There were no significant 3-way or 2-way interactions (ANCOVA) for maximum angle, minimum angle, or ROM at either the stifle or hock. In all analyses no main effect was detected for individual trials or side of the body. Therefore data presented below are the average for two trials from the right and left sides. Gait speed was used as a covariate in all analyses. Fig. 2 presents stifle and hock angles for both sides in all dogs. These results indicate that although the general pattern of motion for GRMD dogs appears similar to their carrier controls, greater variability, as evidenced by the larger SD in the affected dogs, is generally present in the GRMD pattern of motion. The mean within dog CVs for affected and unaffected dogs, respectively, were: maximum stifle angle (4.7\% vs. $2.6 \%, \mathrm{NS})$; minimum stifle angle (5.2\% vs. $4.8 \%$, NS); maximum hock angle ( $7.4 \%$ vs. $3.4 \%, p<0.05$ ); and minimum hock angle ( $7.0 \%$ vs. $5.2 \%$, NS). These data suggest that the variability in the GRMD dogs is greater, consistent with our observations of the SDs in Fig. 2. However, only the $\mathrm{CV}$ for maximum hock angle was significantly different between the groups.

\subsection{Stifle measurements}

The maximum stifle angle in GRMD dogs was larger than the controls ( $136.5 \pm 6.0$ vs. $129.8 \pm 5.7^{\circ}$, respectively, $p=0.037$ ). The minimum stifle angle in GRMD dogs was also larger than the controls (101.6 \pm 6.7 vs. $91.8 \pm 5.1^{\circ}$, respectively, $\left.p=0.019\right)$. However, no differences were detected in the ROM between groups ( $34.9 \pm 6.4$ vs. $38.1 \pm 4.8^{\circ}$, respectively, NS). Together, these data indicate that compared to controls, GRMD dogs walk with their pelvic limb relatively more extended at the stifle throughout the gait cycle.

\subsection{Hock measurements}

There was no difference between the groups in the maximum hock angle ( $138.1 \pm 8.8$ vs. $135.5 \pm 5.8^{\circ}$, respectively, NS). However, the minimum hock angle in GRMD dogs was larger than the controls ( $114.6 \pm 6.5$ vs. $101.8 \pm 7.6^{\circ}$, respectively, $\left.p=0.014\right)$. GRMD dogs also displayed a smaller ROM at this joint compared to controls $\left(23.5 \pm 7.2\right.$ vs. $33.7 \pm 7.7^{\circ}$, respectively, $p=0.067$ ). Further, GRMD dogs demonstrated relatively less flexion at initiation of the gait cycle compared to controls. Together, these findings indicate that compared to controls, GRMD dogs walk with not only less flexion but also less total excursion of the hock joint.

\section{Discussion}

This is the first investigation of gait kinematics focused on the stifle and hock joints of GRMD dogs. The main findings of the present study indicate that: (1) GRMD dogs walked slower than controls; (2) at the stifle joint, both groups displayed similar ROM, but compared to controls GRMD dogs walked with the stifle joint relatively more extended; and (3) at the hock joint, GRMD dogs displayed less ROM and walked with the joint relatively less flexed compared to controls. Since we used gait speed as a covariate in all analyses, the differences we observed in joint kinematics between groups cannot be attributed solely to the slower walking speed of the GRMD dogs.

In DMD patients, the lack of knee flexion during weight acceptance phase of the gait cycle is generally attributed to muscle weakness [22-24]. Because GRMD dogs are weaker than 
normal controls [25], we speculate that affected dogs make adaptations analogous to gait adaptations observed in DMD patients. As a result, GRMD dogs choose a pelvic limb configuration that is more extended but manifest less ROM, and as a consequence, walk more slowly than normal. The observed decrease in hock ROM is in keeping with the static postural characteristics, e.g. contractures, we previously described at this joint [25-27]. However, in our earlier studies the hock angle was more acute than normal.

Importantly, our findings must be interpreted in the context of phenotypic variation typically seen in GRMD. All dogs of this study were over 17 months of age and mildly affected. In more severely affected dogs, the tuber ischii of the pelvis deviate ventrally [28]. As a result, the pelvis shifts forward in a plantigrade position [29]. GRMD dogs with this more severe phenotype would be expected to have more acute hock angles. Considering that proximal muscle imbalance contributes to distal joint contractures in DMD [30], measurement of hip angles might have identified additional differences between GRMD and carrier dogs. The increased variability we observed in the joint angle data (Fig. 2) and the trends for larger within dog CVs for the maximum and minimum angles for the stifle and hock are also likely related to the well-established phenotypic variation seen in affected dogs. We have routinely seen greater variability for joint angles and muscle kinetic parameters in affected versus normal dogs particularly at 6 months of age [25,31].

In general, the patterns we observed in joint kinematics in GRMD dogs were similar to previous reports in normal dogs [12-15,32,33]. The maximum and minimum angles and the ROM at the stifle and hock were identical to those reported by Marsolais and colleagues for a slow walk [19]. In contrast, the ROM we observed in GRMD dogs is less than the ROM reported for dogs with faster walking speeds [13]. Our results are in agreement with those reported by Gillette and Zebas [13] suggesting that canine gait is symmetric at the stifle and hock joints as we found no significant differences between the left and right side gait parameters in either GRMD or carrier dogs.

There are several strengths to the current study. First, we were able to compare the GRMD dogs to their carrier littermates as opposed to a group of "normal" dogs that may have differed in size or breed. Previous studies have indicated that gait may vary among dog breeds $[9,11]$. Second, our data indicate that multiple trials from the same side are similar, and that the right and left side kinematics are also similar within each dog.

The data presented in this study should also be considered in light of several limitations. First, we did not use a constant gait speed for all dogs. Given the influence of speed on gait kinematics $[10,34]$ we examined the feasibility of walking the GRMD dogs on a treadmill to control gait speed during filming. However, the dogs had no previous exposure to the treadmill and we quickly determined that this was not feasible in these dogs due to the clinical manifestations of the disease. While it may be possible to habituate dogs to the treadmill in future studies, the process of treadmill habituation may lead to a training effect which would be a confounding factor. Interestingly, in humans, differences between overground and treadmill walking are small but measurable [35-39]. The majority of studies [17-19,33], but not all [16], suggest that dogs can be habituated to a treadmill in the same manner as humans, [40-42]. However, self-selected walking speed is a relevant outcome because it is an important prognostic indicator of morbidity and mortality [43]. Future work might include both the self-selected speed as well as a controlled treadmill speed.

A second limitation to the present study is that we did not assess the dogs walking on different surfaces. All the data collection in the present study occurred on the same day in the same environment on a concrete surface with a relatively low coefficient of friction. Although walking surface may influence gait kinematics, Kapatkin and her colleagues [44] 
reported that there were no significant differences in ground reaction forces between a linoleum and a carpet surface for thoracic or pelvic limbs. We believe it is unlikely that a different ground surface condition, e.g. carpet, would have substantially modified the differences we observed between the GRMD dogs and their carrier littermates.

Thirdly, the use of surface markers to estimate the location of joint centers may have reduced the accuracy of our joint angle calculations $[45,46]$. While it is possible to implant devices to more accurately assess movement of the bones and joints [47], such invasive measures may have unintended effects on gait kinematics.

Finally, use of two-dimensional versus three-dimensional analysis may be a limitation of the present study. While it is clear that human and canine locomotion occurs in three dimensions [48-50], kinematic analysis with two-dimensional methods is generally considered both accurate and valid for assessment of canine gait $[13,19,48]$.

In summary, this is the first study to measure and compare joint angles at the stifle and hock of GRMD dogs and their age-matched carrier littermates. Our results indicate that, at a selfselected walking pace, compared to normal controls, GRMD dogs walk slower, maintain the stifle joint in a relatively extended position, and exhibit less hock ROM. Methods reported here might be useful in pre-clinical trials using the GRMD canine model.

\section{Acknowledgments}

The authors would like to acknowledge the contributions of Dan Bogan and Janet Bogan for technical assistance and handling of the dogs and Melissa Daly for assistance with programming.

This study was funded by the Wake Forest University Translational Science Institute.

\section{References}

1. Emery AE. Population frequencies of inherited neuromuscular diseases - a world survey. Neuromuscul Disord. 1991; 1(1):19-29. [PubMed: 1822774]

2. Banks GB, Chamberlain JS. The value of mammalian models for Duchenne muscular dystrophy in developing therapeutic strategies. Curr Top Dev Biol. 2008; 84:431-53. [PubMed: 19186250]

3. Collins CA, Morgan JE. Duchenne's muscular dystrophy: animal models used to investigate pathogenesis and develop therapeutic strategies. Int J Exp Pathol. 2003; 84(4):165-72. [PubMed: 14632630]

4. Fletcher S, Ly T, Duff RM, Mcc HJ, Wilton SD. Cryptic splicing involving the splice site mutation in the Canine model of Duchenne muscular dystrophy. Neuromuscul Disord. 2001; 11(3):239-43. [PubMed: 11297938]

5. Howell JM, Fletcher S, Kakulas BA, O'hara M, Lochmuller H, Karpati G. Use of the Dog model for Duchenne muscular dystrophy in gene therapy trials. Neuromuscul Disord. 1997; 7(5):325-8. [PubMed: 9267846]

6. Schatzberg SJ, Anderson LV, Wilton SD, Kornegay JN, Mann CJ, Solomon GG, et al. Alternative dystrophin gene transcripts in golden retriever muscular dystrophy. Muscle Nerve. 1998; 21(8): 991-8. [PubMed: 9655116]

7. Sharp NJ, Kornegay JN, Van Camp SD, Herbstreith MH, Secore S1, Kettle S, et al. An error in dystrophin mRNA processing in golden retriever muscular dystrophy, an animal homologue of Duchenne muscular dystrophy. Genomics. 1992; 13(1):115-21. [PubMed: 1577476]

8. Gillette RL, Angle TC. Recent developments in canine locomotor analysis: a review. Vet J. 2008; 178(2):165-76. [PubMed: 18406641]

9. Bertram JE, Lee DV, Case HN, Todhunter RJ. Comparison of the trotting gaits of labrador retrievers and greyhounds. Am J Vet Res. 2000; 61(7):832-8. [PubMed: 10895909]

10. Blaszczyk JW, Dobrzecka C. Speed control in quadrupedal locomotion: principles of limb coordination in the dog. Acta Neurobiol Exp (Wars). 1989; 49(2-3):105-24. [PubMed: 2728931] 
11. Colborne GR, Innes JF, Comerford EJ, Owen MR, Fuller CJ. Distribution of power across the hind limb joints in labrador retrievers and greyhounds. Am J Vet Res. 2005; 66(9):1563-71. [PubMed: 16261830]

12. Colborne GR, Walker AM, Tattersall AJ, Fuller CJ. Effect of trotting velocity on work patterns of the hind limbs of greyhounds. Am J Vet Res. 2006; 67(8):1293-8. [PubMed: 16881839]

13. Gillette RL, Zebas CJ. A two-dimensional analysis of limb symmetry in the trot of labrador retrievers. J Am Anim Hosp Assoc. 1999; 35(6):515-20. [PubMed: 10580913]

14. Hottinger HA, Decamp CE, Olivier NB, Hauptman JG, Soutas-Little RW. Noninvasive kinematic analysis of the walk in healthy large-breed dogs. Am J Vet Res. 1996; 57(3):381-8. [PubMed: 8669773]

15. Lee JY, Kim G, Kim JH, Choi SH. Kinematic gait analysis of the hind limb after tibial plateau levelling osteotomy and cranial tibial wedge osteotomy in ten dogs. J Vet Med A Physiol Pathol Clin Med. 2007; 54(10):579-84. [PubMed: 18045343]

16. Clements DN, Owen MR, Carmichael S, Reid SW. Kinematic analysis of the gait of 10 labrador retrievers during treadmill locomotion. Vet Rec. 2005; 156(15):478-81. [PubMed: 15828744]

17. Fanchon L, Valette JP, Sanaa M, Grandjean D. The measurement of ground reaction force in dogs trotting on a treadmill: an investigation of habituation. Vet Comp Orthop Traumatol. 2006; 19(2): 81-6. [PubMed: 16810349]

18. Fanchon L, Grandjean D. Habituation of healthy dogs to treadmill trotting: repeatability assessment of vertical ground reaction force. Res Vet Sci. 2008

19. Marsolais GS, Mclean S, Derrick T, Conzemius MG. Kinematic analysis of the hind limb during swimming and walking in healthy dogs and dogs with surgically corrected cranial cruciate ligament rupture. J Am Vet Med Assoc. 2003; 222(6):739-43. [PubMed: 12675295]

20. Vilensky JA, O'connor BL, Brandt KD, Dunn EA, Rogers PI, Delong CA. Serial kinematic analysis of the unstable knee after transection of the anterior cruciate ligament: temporal and angular changes in a canine model of osteoarthritis. J Orthop Res. 1994; 12(2):229-37. [PubMed: 8164096]

21. Bartlett RJ, Winand NJ, Secore SL, Singer JT, Fletcher S, Wilton S, et al. Mutation segregation and rapid carrier detection of X-linked muscular dystrophy in dogs. Am J Vet Res. 1996; 57(5): 650-4. [PubMed: 8723876]

22. Armand S, Mercier M, Watelain E, Patte K, Pelissier J, Rivier F. A comparison of gait in spinal muscular atrophy, type II and Duchenne muscular dystrophy. Gait Posture. 2005; 21(4):369-78. [PubMed: 15886126]

23. D'angelo MG, Berti M, Piccinini L, Romei M, Guglieri M, Bonato S, et al. Gait pattern in Duchenne muscular dystrophy. Gait Posture. 2009; 29(1):36-41. [PubMed: 18656361]

24. Sussman M. Duchenne muscular dystrophy. J Am Acad Orthop Surg. 2002; 10(2):138-51. [PubMed: 11929208]

25. Kornegay JN, Bogan DJ, Bogan JR, Childers MK, Cundiff DD, Petroski GF, et al. Contraction force generated by tarsal joint flexion and extension in dogs with golden retriever muscular dystrophy. J Neurol Sci. 1999; 166(2):115-21. [PubMed: 10475104]

26. Childers MK, Staley JT, Kornegay JN, Mcdonald KS. Skinned single fibers from normal and dystrophin-deficient dogs incur comparable stretch-induced force deficits. Muscle Nerve. 2005; 31(6):768-71. [PubMed: 15736272]

27. Kornegay JN, Sharp NJ, Bogan DJ, Van Camp SD, Metcalf JR, Schueler RO. Contraction tension and kinetics of the peroneus longus muscle in golden retriever muscular dystrophy. J Neurol Sci. 1994; 123(1-2):100-7. [PubMed: 8064300]

28. Brumitt JW, Essman SC, Kornegay JN, Graham JP, Weber WJ, Berry CR. Radiographic features of golden retriever muscular dystrophy. Vet Radiol Ultrasound. 2006; 47(6):574-80. [PubMed: 17153067]

29. Valentine BA, Cooper BJ, De LA, O'quinn R, Blue JT. Canine X-linked muscular dystrophy. An animal model of Duchenne muscular dystrophy: clinical studies. J Neurol Sci. 1988; 88(1-3):6981. [PubMed: 3225630]

30. Vignos PJ Jr, Archibald KC. Maintenance of ambulation in childhood muscular dystrophy. J Chronic Dis. 1960; 12:273-90. [PubMed: 13842210] 
31. Kornegay JN, Sharp NJ, Schueler RO, Betts CW. Tarsal joint contracture in dogs with golden retriever muscular dystrophy. Lab Anim Sci. 1994; 44(4):331-3. [PubMed: 7983843]

32. Decamp CE, Soutas-Little RW, Hauptman J, Olivier B, Braden T, Walton A. Kinematic gait analysis of the trot in healthy greyhounds. Am J Vet Res. 1993; 54(4):627-34. [PubMed: 8484586]

33. Owen MR, Richards JG, Clements DN, Drew ST, Bennett D, Carmichael S. Kinematics of the elbow and stifle joints in greyhounds during treadmill trotting —an investigation of familiarisation. Vet Comp Orthop Traumatol. 2004; 17:141-5.

34. Maes LD, Herbin M, Hackert R, Bels VL, Abourachid A. Steady locomotion in dogs: temporal and associated spatial coordination patterns and the effect of speed. J Exp Biol. 2008; 211(Pt 1):13849. [PubMed: 18083742]

35. Herbin M, Hackert R, Gasc JP, Renous S. Gait parameters of treadmill versus overground locomotion in mouse. Behav Brain Res. 2007; 181(2):173-9. [PubMed: 17521749]

36. Nelson RC, Dillman CJ, Lagasse P, Bickett P. Biomechanics of overground versus treadmill running. Med Sci Sports. 1972; 4(4):233-40. [PubMed: 4648586]

37. Pereira JE, Cabrita AM, Filipe VM, Bulas-Cruz J, Couto PA, Melo-Pinto P, et al. A comparison analysis of hindlimb kinematics during overground and treadmill locomotion in rats. Behav Brain Res. 2006; 172(2):212-8. [PubMed: 16777243]

38. Riley PO, Paolini G, Della CU, Paylo KW, Kerrigan DC. A kinematic and kinetic comparison of overground and treadmill walking in healthy subjects. Gait Posture. 2007; 26(1):17-24. [PubMed: 16905322]

39. Riley PO, Dicharry J, Franz J, Croce UD, Wilder RP, Kerrigan DC. A kinematics and kinetic comparison of overground and treadmill running. Med Sci Sports Exerc. 2008; 40(6):1093-100. [PubMed: 18460996]

40. Charteris J, Taves C. The process of habituation to treadmill walking: a kinematic analysis. Percept Mot Skills. 1978; 47(2):659-66. [PubMed: 724408]

41. Van De Putte M, Hagemeister N, St-Onge N, Parent G, De Guise JA. Habituation to treadmill walking. Biomed Mater Eng. 2006; 16(1):43-52. [PubMed: 16410643]

42. Wall JC, Charteris J. The process of habituation to treadmill walking at different velocities. Ergonomics. 1980; 23(5):425-35. [PubMed: 7202394]

43. Guralnik JM, Ferrucci L, Pieper CF, Leveille SG, Markides KS, Ostir GV, et al. Lower extremity function and subsequent disability: consistency across studies, predictive models, and value of gait speed alone compared with the short physical performance battery. J Gerontol A Biol Sci Med Sci. 2000; 55(4):M221-31. [PubMed: 10811152]

44. Kapatkin AS, Arbittier G, Kass PH, Gilley RS, Smith GK. Kinetic gait analysis of healthy dogs on two different surfaces. Vet Surg. 2007; 36(6):605-8. [PubMed: 17686136]

45. Audigie F, Pourcelot P, Degueurce C, Denoix JM, Geiger D, Bortolussi C. Asymmetry in placement of bilateral skin markers on horses and effects of asymmetric skin marker placement on kinematic variables. Am J Vet Res. 1998; 59(8):938-44. [PubMed: 9706195]

46. Lucchetti L, Cappozzo A, Cappello A, Della CU. Skin movement artifact assessment and compensation in the estimation of knee-joint kinematics. J Biomech. 1998; 31(11):977-84. [PubMed: 9880054]

47. Korvick DL, Pijanowski GJ, Schaeffer DJ. Three-dimensional kinematics of the intact and cranial cruciate ligament-deficient stifle of dogs. J Biomech. 1994; 27(1):77-87. [PubMed: 8106538]

48. Kim J, Rietdyk S, Breur GJ. Comparison of two-dimensional and three-dimensional systems for kinematic analysis of the sagittal motion of canine hind limbs during walking. Am J Vet Res. 2008; 69(9):1116-22. [PubMed: 18764680]

49. Ounpuu S. The biomechanics of walking and running. Clin Sports Med. 1994; 13(4):843-63. [PubMed: 7805110]

50. Tashman S, Anderst W, Kolowich P, Havstad S, Arnoczky S. Kinematics of the Acl-deficient canine knee during gait: serial changes over two years. J Orthop Res. 2004; 22(5):931-41. [PubMed: 15304262] 


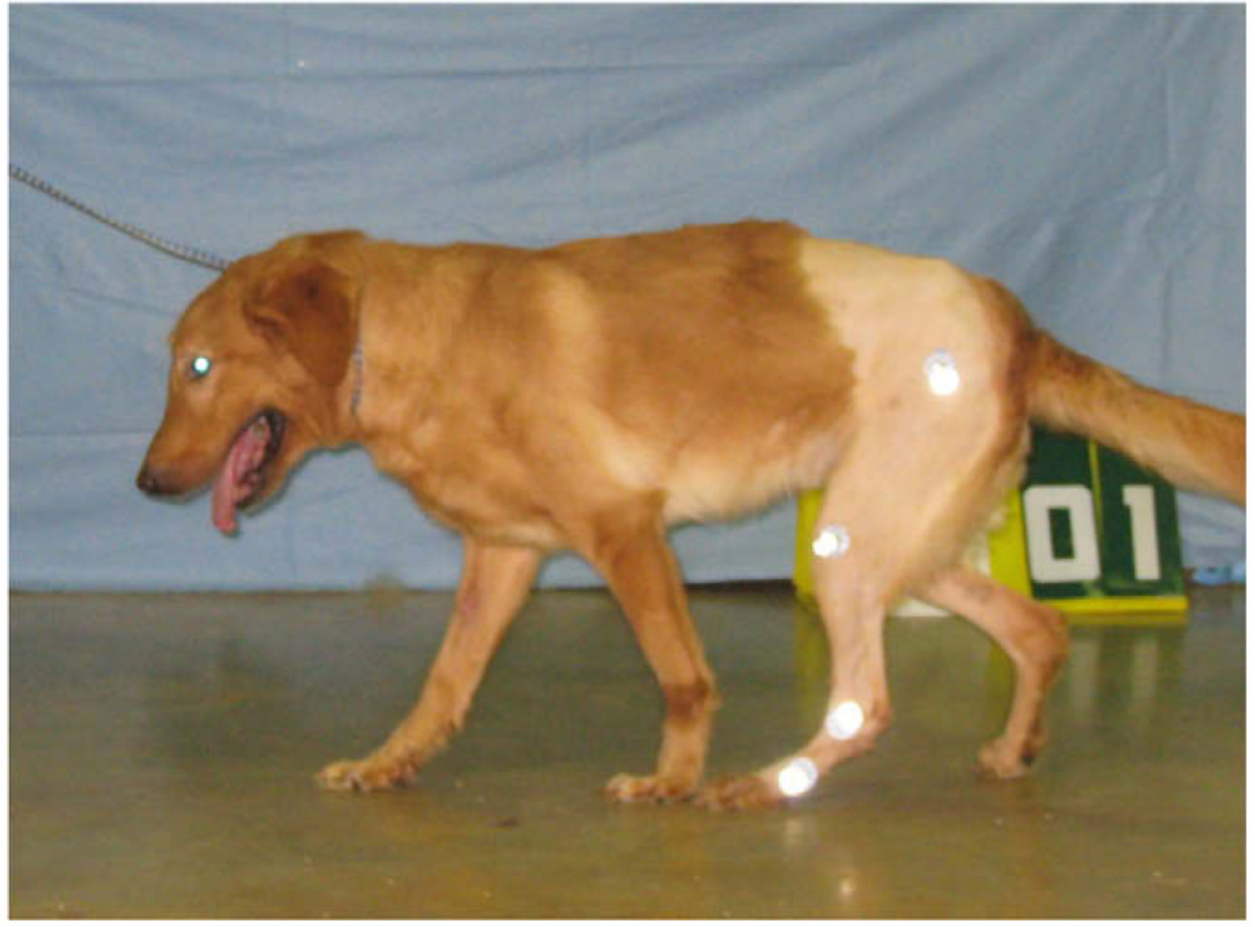

Fig. 1.

Filming environment showing joint marker placement on a GRMD dog. 

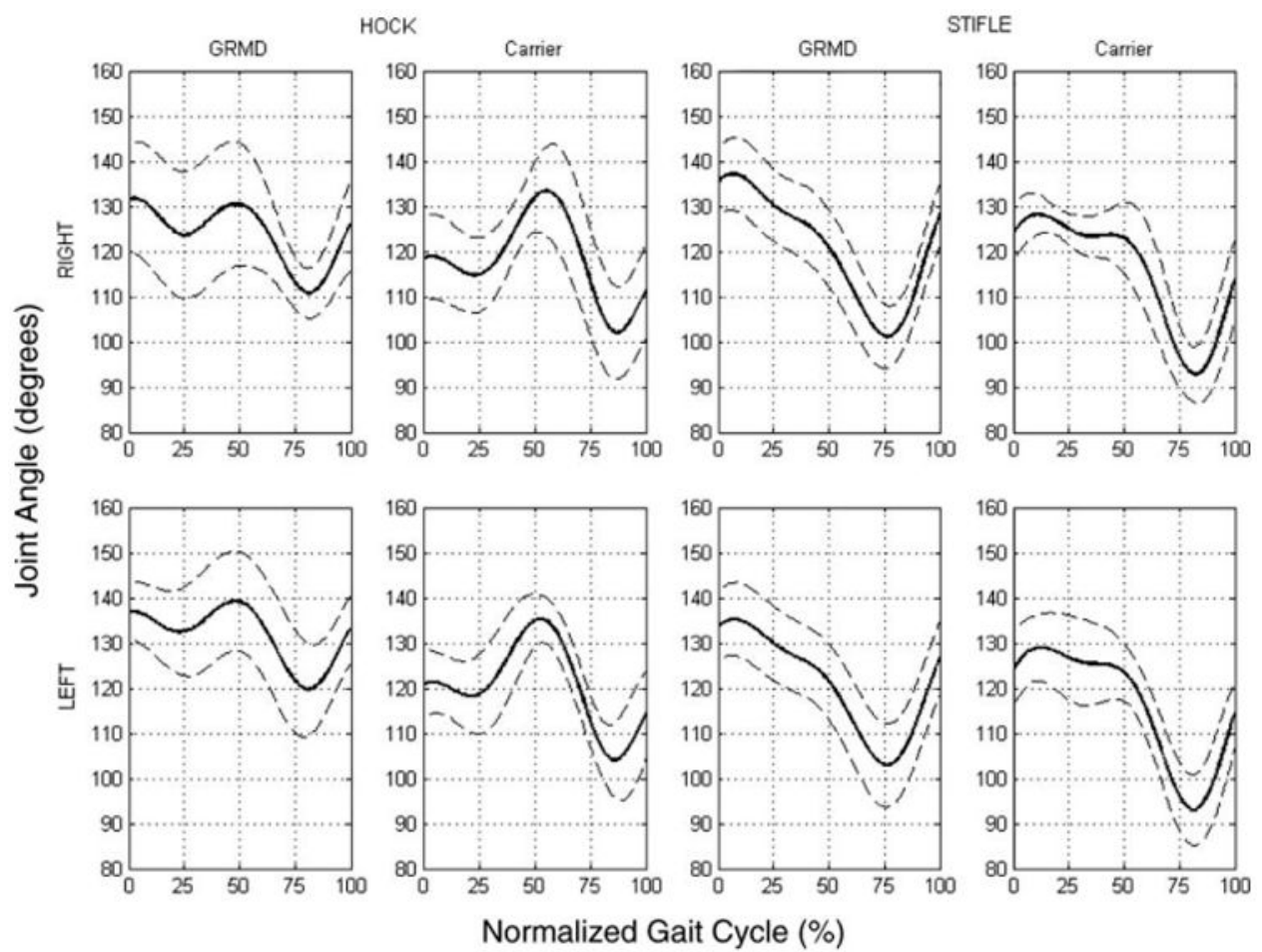

Fig. 2.

Joint angle data for the stifle and hock of GRMD and carrier dogs. The $x$-axis represents the gait cycle from heel strike to heel strike. The y-axis represents joint angle in degrees. The solid line is the mean and the dotted line is $1 \mathrm{SD}$. 
Table 1

Characteristics of GRMD and age-matched carrier dogs.

\begin{tabular}{lllll}
\hline Dog & Gender & Age $(\mathbf{m o s})$ & Shoulder height $(\mathbf{c m})$ & Body mass $(\mathbf{k g})$ \\
\hline GRMD & & & & \\
Gus & M & 17.25 & 43 & 11.7 \\
Mozart & M & 41.5 & 50 & 19 \\
Satchmo & M & 20.75 & 47 & 16.6 \\
Chuckie & M & 21.25 & 50 & 19.5 \\
Pictor & M & 32.5 & 49 & 20.6 \\
Omi & M & 21.5 & 55 & 20.4 \\
Mean & & 25.8 & 49.0 & 18.0 \\
SD & & 9.3 & 3.9 & 3.4 \\
Carriers & & & & \\
Anastasia & F & 17.25 & 47 & 21 \\
Tchaikovsky & F & 41.5 & 46 & 23.4 \\
Lena & F & 20.75 & 48 & 17 \\
Smurfette & F & 22.5 & 42 & 17.4 \\
Vela & F & 32.5 & 46 & 21.7 \\
Samara & F & 21.25 & 44 & 16.2 \\
Mean & & 26.0 & 45.5 & 19.5 \\
SD & & 9.2 & 2.2 & 3.0 \\
\hline & & & & \\
\hline
\end{tabular}

